\title{
Image Processing Techniques of Thermal Wave Acquired from Application of Infrared Thermography in Detecting the Subsurface Defects of the \\ Material
}

\author{
Wontae $\mathrm{Kim}^{1}$, Shrestha Ranjit ${ }^{1}$ and Manyong $\mathrm{Choi}^{2}$ \\ ${ }^{1}$ Kongju National University, 1223-24 Cheoan Daero, Seobuk-gu, Chungnam, Korea \\ ${ }^{2}$ Korea Research Institute of Standards and Science, 1 Doryong-dong, Yuseong-gu, Daejeon, \\ Korea \\ Email:kwt@kongju.ac.kr
}

\begin{abstract}
Since infrared thermography is an emerging approach with non-contact, non-intrusive, and non-destructive, it was capable to be applied as one of inspections of various solid materials such as metals, composites and semiconductors for industrial. And research interests were widely conducted with the objective of quantitative determination of the sizes and locations of subsurface defects. In this study, image processing was applied to infrared thermography images to detect defects in metals that were widely used in industrial fields. $t$.
\end{abstract}

Keywords: Thermal wave, image processing, subsurface, infrared thermography, defects

\section{Introduction}

The response of the material to the thermal stimulus is dependent on the existence of subsurface defects and their features. In order to obtain the information about defects, the material's response to the thermal stimulus is studied. In this research, the study has been done on three dimensional solid bodies with artificially created subsurface defects at some depth to simulate the heat flow in the infrared thermography inspection process.

\section{Theory}

In lock-in thermography, after externally heating the specimen sinusoidally (with fixed amplitude and frequency), the resultant temperature distribution on the surface is observed in the stationary regime and the corresponding data is recorded real time. The amplitude image displays the total temperature increase on a specimen during power cycling and is used to determine fault location. Phase represents the delay between device powering and the resulting device heating and used to calculate the defect depth of the fault. For example, if $\mathrm{S} 1, \mathrm{~S} 2, \mathrm{~S} 3$ and S4 are four equidistant temperature data points (as shown in Fig. 1.) in a complete period then the phase $(\varnothing)$ and amplitude $(\mathrm{A})$ are given by, 


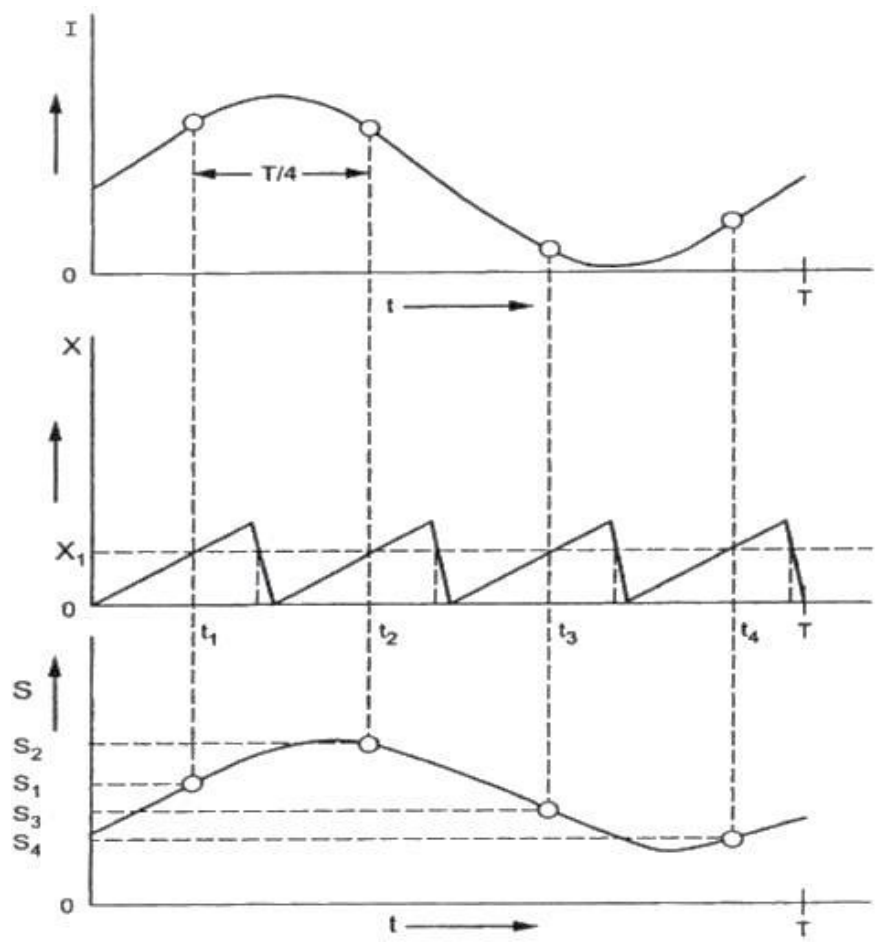

Figure 1. Principle of computation of phase, amplitude and thermographic images in lock-in thermography

\section{Data Processing Methodology}

For generation of sine waves of a single frequency, a programmable function generator, (Agilent 33210A, Malaysia) was used and for detection of thermal waves infrared camera (SC645, FLIR Systems, Sweden) was used that has a 640×240 pixel resolution and sensitivity of 7.5-13 $\mu \mathrm{m}$. When analyzing experimental data from infrared thermographic testing, raw images were often not appropriate. Thus, various image processing methods were applied at the pre-processing and processing levels in image processing program (MATLAB) for quantitative evaluation of defect detection and characterization which increased the infrared non-destructive testing capabilities since subtle defects signature became apparen

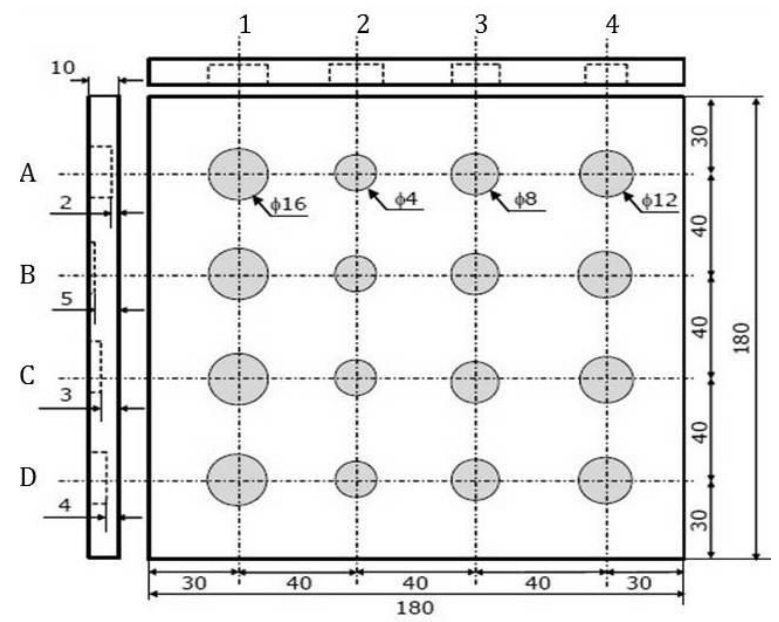

Figure 2. Inclusion defect specimen of STS 304 


\section{Results and Discussions}

The thermographic thermal images at $\mathrm{t}=8.24 \mathrm{~s}, 9.62 \mathrm{~s}, 10.99 \mathrm{~s}$ and $12.36 \mathrm{~s}$, amplitude image and phase images are shown in Fig. 3.

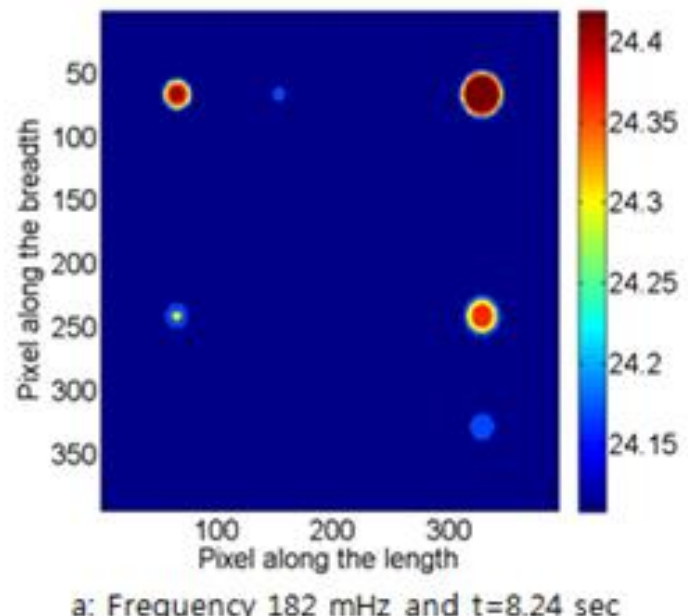

Figure 3. Thermographic Amplitude and Phase Imaging at frequency of $182 \mathrm{mHz}$.

\section{Conclusion}

In this work, various defect detection and quantitative characterization methods were applied along with the image processing for the defect detections and found good resolution using $M A T L A B$ as one of image processing techniques.

\section{References}

[1] H. Czichos, 'Handbook of Technical Diagnostics, Fundamentals and Application to Structures and Systems', pp.175-220, 2013

[2] X.P.V. Maldague, Lock-in Thermography, 'Theory and practice of infrared technology for non-destructive testing', pp. 355-362, 2001

[3] X.P.V. Maldague and P.O. Moore, 'Lock-in Thermography, Non-destructive Testing Handbook', Third Edition, Vol.3, pp. 318-327, 2001 